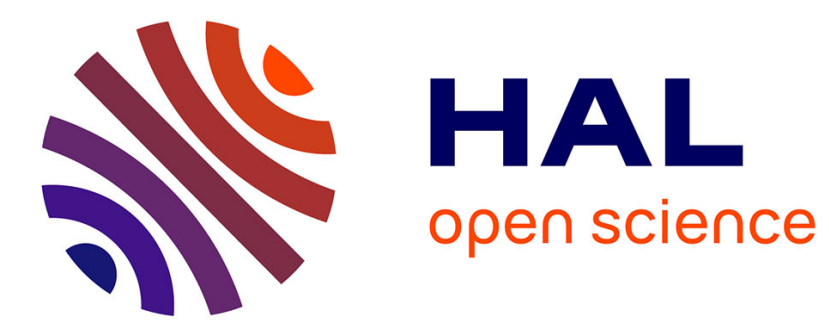

\title{
Reformulation of the coupled problem for the simplified closed channel
}

Daniele Tomatis

\section{To cite this version:}

Daniele Tomatis. Reformulation of the coupled problem for the simplified closed channel. Annals of Nuclear Energy, 2019, 130, pp.377-387. 10.1016/j.anucene.2019.03.001 . cea-02510805

\section{HAL Id: cea-02510805 https://hal-cea.archives-ouvertes.fr/cea-02510805}

Submitted on 18 Mar 2020

HAL is a multi-disciplinary open access archive for the deposit and dissemination of scientific research documents, whether they are published or not. The documents may come from teaching and research institutions in France or abroad, or from public or private research centers.
L'archive ouverte pluridisciplinaire HAL, est destinée au dépôt et à la diffusion de documents scientifiques de niveau recherche, publiés ou non, émanant des établissements d'enseignement et de recherche français ou étrangers, des laboratoires publics ou privés. 


\title{
Reformulation of the coupled problem in the simplified closed channel
}

\author{
Daniele Tomatis* \\ DEN/DANS/DM2S/SERMA/LPEC, \\ Service d'études des réacteurs et de mathématiques appliquées, \\ CEA, Université Paris-Saclay, F-91191 Gif-sur-Yvette, France
}

$13 / 09 / 2016$

\begin{abstract}
Several types of nuclear reactors rely on lattices of fuel rods, where the fuel cells constitute the basic units of energy production by the nuclear reactions and of energy removal by the flowing coolant. The coolant channel is physically identified by the volume within adjacent fuel cells, being the fundamental purview of design and safety studies resolving coupled thermo-hydraulic and neutron problems. Although channels are generally opened for optimal heat transfer, its closed version is often in use to describe representative channels in boxed fuel assemblies, or simply faster predictive modelling. This work aims to find a solution to the coupled closed channel problem without solving separately for the different physics in an iterative scheme. The new methodology developed hereafter is demonstrated on a realistic PWR channel with UO2 fuel, and compared against the traditional one based on operator-splitting. The simple model presented in this work serves both research and educational purposes.
\end{abstract}

Keywords - Multiphysics coupling, closed channel, PWR.

${ }^{*}$ Corresponding author: daniele.tomatis@cea.fr 


\section{Contents}

1 Introduction $\quad 2$

2 The closed channel model $\quad 3$

3 One-group neutron diffusion $\quad 4$

3.1 Reformulation for the coupling ................ 5

4 Heat transfer in the fuel cell $\quad 6$

5 Numerical results $\quad \mathbf{7}$

5.1 Preparation of nuclear data . . . . . . . . . . . . . . . 8

5.2 Numerical solution of the non-linear problem . . . . . . . . . . . . 11

6 Conclusion

A Simplified model without Doppler feedback

\section{Introduction}

The determination of the thermal power generated in a nuclear reactor is a coupled multiphysics problem, involving neutron physics and thermal-hydraulic models. The solution of the whole problem is generally addressed by separate linearized problems coupled through successive iterations up to a fixed point [1].

The nuclear reactor is modelled as a compact structure of connected regions, where the geometry is usually Cartesian and regular, and coarse meshes are employed. The computational cells are filled with homogenized material data. Here, the neutron solver computes the thermal power from fission provided a distribution of input nuclear cross sections, whose homogenization has already been prepared and validated. Next, the thermal power is used to feed a thermal-hydraulic model in order to determine maps of thermo-dynamics and fluid properties in the core. Homogenized cross sections are modelled as dependent on a few thermal-hydraulic properties to reproduce the physical feedback on the neutron reactivity. Hence, the new maps of thermal-hydraulic properties are used to update cross sections and to start new neutron calculations. This iterative process is continued up to convergence on all unknowns of the problem: the neutron flux and cross sections yielding the reaction rates, and all maps of thermal-hydraulic properties considered for the cross section update.

The setup of this kind of schemes in real reactor problems is in general cumbersome due to possible stability issues and to the sensitivity to the initialization of the unknowns. Successive relation with parameters less than $1 / 2$ can be necessary requesting many expensive calculations with several calls to the different physical solvers. It must be noted that the majority of the core simulators works with schemes based on successive iterations. Steady state problems are typical of design studies, whereas transients are mostly dedicated to safety in both accidental and operational conditions.

The goal of this article is to present an alternative methodology to solve the coupled problem, possibly avoiding linearization and combination of separate physical solutions. The problem is firstly restrained to the simpler closed channel model without friction and in steady state conditions with this preliminary work. The heat transfer model presented here 
covers the PWR physics only, postponing to later works the extension of the methodology to other reactor types.

The interest in this topic follows after recent works by Dellacherie et Al. [2, 3, 4], who first addressed the problem under a mathematical point of view. However, their physical assumptions limit the applications in real problems. Specifically, the numerical case presented by Dellacherie in [4] assumes implicitly prior knowledge about the solution of the coupled problem by providing nuclear data as functions of the only coolant enthalpy. Our approach is different in the form and keeps the full non-linearity of the problem. Neutron diffusion is used with the one-group energy formalism. A numerical demonstration of the solution is analyzed in a realistic engineering case.

\section{The closed channel model}

The closed channel model is a simple thermal-hydraulic model which basically considers only the enthalpy rise of the coolant in the channel along the direction of flow [5]. This model is suited to represent boxed fuel assemblies as in BWRs. Energy, mass and momentum transfers on the transverse motion directions of the coolant flow are neglected. This model is the starting point to build more realistic and more complex models.

The mass-specific enthalpy $h(\mathrm{~J} / \mathrm{kg})$ of the flow moving through the channel is determined by the energy balance in an infinitesimal axial element $d z$ :

$$
q d_{z} h=P_{l}
$$

where $q$ is the mass flow rate of the coolant $(\mathrm{g} / \mathrm{s})$ and $P_{l}$ is the linear heat rate $(\mathrm{W} / \mathrm{cm})$ generated by fissions. A closed channel implies also mass conservation with a constant $q$ all along the channel: $q=u \varrho A_{c}$ with $u$ as the coolant speed $(\mathrm{cm} / \mathrm{s}), \varrho$ as the coolant density $\left(\mathrm{g} / \mathrm{cm}^{3}\right)$ and $A_{c}$ as the wetted area of the channel $\left(\mathrm{cm}^{2}\right)$. Given a physical channel of cell pitch $l_{c}, A_{c}=l_{c}^{2}-\pi r_{c, \text { out }}$, where $r_{c, \text { out }}$ is the outer clad surface radius.

Once the thermo-dynamical state of the coolant is known, the coolant speed $u$ can be calculated at any position $z$ since $d_{z} q=0$. Similarly, the dynamic pressure $\varpi$ follows after the integration of the momentum equation:

$$
d_{z}\left(\varrho u^{2}+\varpi\right)=\varrho g,
$$

with the volume-specific gravity force at RHS. This model is also referred as low Mach number model by Dellacherie et Al. [2].

$P_{l}$ is determined in cylindrical polar coordinates as:

$$
P_{l}(z)=\int_{0}^{\infty} d E \int_{S_{f}} 2 \pi r d r d \theta\left[\sigma_{\kappa} \phi\right](\theta, r, E),
$$

with $S_{f}$ as the fuel heating area, $\sigma_{\kappa}$ as the energy production cross section $(\mathrm{J} / \mathrm{cm}), \phi$ as the neutron flux (particles $/ \mathrm{cm}^{2} / \mathrm{s}$ ). The integration in energy accounts for contributions of neutrons traveling at any speed. $P_{l}$ is determined by the sum of the heat flux at the outer cladding surface and the power deposited in the coolant by gamma radiation. For stationarity, the energy produced in the fuel is directly transferred to the fluid at each axial position by means of the heat flux $\Phi=P_{l} /\left(2 \pi r_{c, \text { out }}\right)\left(\mathrm{W} / \mathrm{cm}^{2}\right)$. Enthalpy at the entrance of the channel is $h_{\mathrm{i}}=h\left(z=z_{i}=0\right)$, whereas at the top it is $h_{\mathrm{O}}=h\left(z=z_{0}=H\right)$. Both, as well as the thermal power produced in the whole channel, i.e. $\mathcal{P}=\int_{0}^{H} P_{l} d z(\mathrm{~W})$, 
are given as input data as customarily used to design the reactor. Besides, the following expression must be fulfilled:

$$
h_{\mathrm{O}}=h_{\mathrm{i}}+\mathcal{P} / q .
$$

It is interesting to notice the integration on all neutron energies in Eq. 2 to obtain the total thermal power which contributes to the enthalpy rise across $d z$ of $q$. This projection suggests to start with one-group theory for the resolution of the neutron problem. The integral operator will be examined later, when treating the common multi-group formalism of the neutron population.

Hence provided one-group nuclear data, the reaction rate of energy production is assumed as proportional to the fission reaction rate hereafter:

$$
P_{l}=\kappa \sigma_{f} \phi
$$

where $\sigma_{f}$ is the one-group fission cross section homogenized in the fuel pin cell and at a given height $z$, and $\kappa$ is the average energy produced per fission $\left(\sigma_{\kappa}=\kappa \sigma_{f}\right)$. The flux $\phi$ is here considered as volume-integrated. Because of Eq. 1 and the positivity of the neutron production, the enthalpy $h$ is a monotone increasing function.

About the neutron balance, we use here the diffusion approximation largely adopted in reactor core physics. Its form will be introduced in section 3 .

\section{One-group neutron diffusion}

The one-group neutron diffusion equation is (using standard notation):

$$
\left[-d_{z} D d_{z}+\sigma_{a}\right] \phi=\lambda \nu \sigma_{f} \phi
$$

with all nuclear data as dependent on the total neutron flux $\phi$ and on the coolant enthalpy $h$ at the axial position $z$. The dependence on the flux sustained by a unique local power level, which changes the fuel temperature indeed, reproduces the thermal Doppler feedback. The dependence on the coolant enthalpy reproduces the moderator effect instead. Because of Eqs. 1 and 4, the flux is here proportional to the first derivative in $z$ of the enthalpy.

The coefficient $\lambda$ ensures the degeneracy of the global operator of Eq. 5, so that a non-trivial solution can be obtained to operate the system at critical conditions. Nuclear engineers are used to introduce an eigenvalue into the general linear form of the Boltzmann equation for neutrons, whenever critical conditions are requested to operate the reactor. But here, $\lambda$ becomes a simple constant parameter leaving the adjustment on degeneracy to the dependence of cross sections on the macroscopic thermo-dynamical properties. This is an important remark. Eq. 5 does not model external neutron sources, but they could be added after providing a sub-critical configuration to the system.

Zero incoming current is used as boundary condition for Eq. 5 by means of extrapolated lengths at the bottom and at the top of the heated channel. This entails

$$
d_{z} \phi= \pm \epsilon^{-1} \phi
$$

respectively at the inlet $z_{i}$ and at the outlet $z_{o}$, with the extrapolation length $\epsilon=2 D$ by diffusion theory [6]. Note also that $\epsilon=0$ can reproduce vanishing flux at the boundary, whereas axial reflection comes with $\epsilon \rightarrow+\infty$. The problem is considered purely monodimensional disregarding any gradient across the neighboring channels. 


\subsection{Reformulation for the coupling}

A new formulation of the problem coupling the one-group neutron diffusion equation, Eq. 5, and the thermal energy balance, Eq. 1, is sought in this section by determining the dependence of the neutron flux on the coolant enthalpy.

Still by means of Eqs. 1 and 4, and using the chain rule to replace the differentiation in $z$ to $h$, we rewrite the neutron balance as:

$$
-\frac{P_{l}}{q} \frac{d}{d h}\left(D \frac{P_{l}}{q} \frac{d \phi}{d h}\right)=\left(\lambda-\frac{1}{k_{\infty}}\right) \nu \sigma_{f} \phi=\left(\rho_{\infty}-\rho\right) \frac{\nu}{\kappa} P_{l} .
$$

The introduction of the static reactivity, i.e. $\rho=1-1 / k$, allows writing $\lambda-k_{\infty}^{-1}=\rho_{\infty}-\rho$, with the multiplication constant in the infinite medium $k_{\infty}=\nu \sigma_{f} / \sigma_{a}$.

After some simplification and provided a constant neutron multiplicity $\nu$, a new second-order ordinary differential equation arises:

$$
-d_{h} \tilde{D} d_{h} \phi^{2}=\zeta\left(\rho_{\infty}-\rho\right)
$$

with the new dimensionless quantity $\tilde{D}=D \sigma_{f}$ and the known constant $\zeta=2 \nu(q / \kappa)^{2}$. The system reactivity $\rho$ remains an unknown constant, instead. Its role is supposed to calibrate the solution in order to satisfy the target power production and removal in the channel within the given active height. In these terms, it is still a critical parameter to operate the system. Although the modifications do not remove non-linearity, the original coupled problem is now contained in a single equation. Remarkably, this is not an eigenvalue equation anymore.

In diffusion $D \approx \lambda_{t} / 3$, where $\lambda_{t}=1 / \sigma_{t}$ is the mean free path of migrating neutrons, equal to the inverse of the total cross section $\sigma_{t}$. $\tilde{D}$ is then proportional to the probability that once a neutron suffers a collision event, this last ends in a fission. As $\rho_{\infty}$, it depends on the local enthalpy and on the flux level, whose same behavior in enthalpy constitutes indeed the solution of the coupled problem.

Eq. 8 must be complemented by new boundary conditions obtained from Eq. 6 . The same chain rule yields (with now $\epsilon=2 D$ ):

$$
\phi d_{h} \phi= \pm \frac{q}{2 \kappa \tilde{D}} \phi
$$

at the channel inlet and outlet, respectively. This becomes $(2 \kappa \tilde{D}) d_{h} \phi= \pm q$ on condition that the flux does not vanish at the boundary. If the flux vanishes instead, there is no power generation at the boundary and the coolant conserves its enthalpy (at least for the infinitesimal $d z$ ) due to $d_{z} h=0$. Since $d_{z} h=d_{z} \phi d_{\phi} h=0$, it follows that $d_{\phi} h=0$ or $d_{h} \phi \rightarrow+\infty$. This warns for a (half) cusp singularity of $\phi(h)$ when approaching the boundary. Finally, reflection writes $d_{z} \phi=0=d_{z} h d_{h} \phi$, requiring $d_{h} \phi=0$ to allow for any possible power value at the boundary.

Eq. 8 is a boundary value problem, generally non-linear and in-homogeneous; linearity can only be attained with specific forms of $D$ and $\rho_{\infty}$, see appendix A for instance. The nuclear data present in Eq. 8 must be functionalized on enthalpy and on the neutron flux. Simple linear approximations are calculated in section 5.1 by means of several independent lattice calculations, where the temperature values in the pin cell are determined by the heat transfer from section 4 . This allows to find the solution as a function of the system reactivity. Sure, any function approximation of the physical data that offers analytical solutions to Eq. 8 is strongly advised. 
Eventually by Eq. 1, the reactivity is determined by constraining the generated thermal power over the given active height $H$, as:

$$
q \int_{h_{\mathrm{i}}}^{h_{\mathrm{O}}} \frac{d h^{\prime}}{P_{l}}=H,
$$

provided that $P_{l}(h)>0$, for $h \in\left(h_{\mathrm{i}}, h_{\mathrm{O}}\right)$. Since our problem assumes that $\sigma_{f}$ never vanishes on the same interval, and that the flux may only become zero on the boundary, Eq. 10 can still be resolved as improper integral, that is taking the limit of the definite integral on the integral bounds. We also note that the same integral equation allows to return to the original problem in the axial coordinate $z$, with the solution $h(z)$ as upper bound of:

$$
z=\frac{q}{\kappa} \int_{h_{0}}^{h} \frac{d h^{\prime}}{\sigma_{f} \phi\left(h^{\prime}\right)} .
$$

Once enthalpy is known at a given position $z$, all other physical data follow accordingly. The change of variable demands the enthalpy to be a strictly increasing function of $z$, that is the power cannot vanish within the domain. Eq. 8 does not hold for regions without fissile materials, like the reflector for instance, where $\sigma_{f}=0$. In such regions, the enthalpy remains unchanged under the formulated hypothesis that no friction or other dissipative forces act on the fluid. Furthermore, different independent variables for the cross section data characterizing these regions may be needed, on a case-dependent base.

\section{Heat transfer in the fuel cell}

The distribution of the power production in the pellet depends on the local thermodynamical state characterizing the response of the matter with the colliding neutrons. Specifically, the fuel temperature and at least two state variables for the (single-phase) coolant are needed to setup the neutron calculation. We use only the coolant enthalpy for the assumed constant pressure along the channel, determining the other quantities like the density by the state functions. Hence, the fuel temperature corresponding to a given linear power and enthalpy of the coolant must be determined.

A simplified thermal-hydraulic model derived from FRAPCON-4.0 is explained in this section to approximate the temperature profile in the fuel pin [7]. This model provides realistic values, avoiding to recur to more sophisticated calculations with the thermalhydraulic and the fuel performance computer codes.

Provided a bulk temperature in the moderator $T_{m}$, the heat transfer in the film washing the clad is reproduced either by forced convection or by nucleate boiling to determine the cladding wall temperature $T_{w}$. The selection between the two regimes follows using the minimum value between $T_{m}+\Delta T_{f}$ and $T_{\text {sat }}+\Delta T_{J L}$, being $T_{\text {sat }}$ the coolant saturation temperature, $\Delta T_{f}$ the forced convection film temperature drop and $\Delta T_{J L}$ the nucleate boiling temperature drop. Temperature are here in $\mathrm{K}$, and the presence of possible crud or oxide layers is neglected for simplicity.

Because of the steady-state condition, the thermal heat flux leaving the rod is analytically determined as $\Phi_{c, \text { out }}=P_{l} /\left(2 \pi r_{c, \text { out }}\right)\left(\mathrm{W} / \mathrm{m}^{2}\right)$, where $r_{c, \text { out }}$ is the outer cladding radius. The forced convection temperature drop is calculated as $\Delta T_{f}=\Phi_{c, \text { out }} / h_{f}$, with the film conductance by the Dittus-Boelter correlation:

$$
h_{f}=2.3 \times 10^{-2} \frac{k}{D_{e}} \operatorname{Re}^{0.8} \operatorname{Pr}^{0.4},
$$


$D_{e}$ is the channel hydraulic diameter $(\mathrm{m})$, whereas Re and Pr are respectively the Reynolds and the Prandtl numbers (dimensionless):

$$
D_{e}=2 \frac{A_{c}}{\pi r_{c, \text { out }}}, \operatorname{Re}=\frac{q D_{e}}{A_{c} \mu} \text { and } \operatorname{Pr}=\frac{c_{p} \mu}{k} .
$$

The other properties are the thermal conductivity of the coolant $k(\mathrm{~W} / \mathrm{m} / \mathrm{K})$, the dynamic viscosity $\mu(\mathrm{Pa} \cdot \mathrm{s})$ and the mass-specific heat $c_{p}(\mathrm{~J} / \mathrm{kg} / \mathrm{K})$.

The temperature drop in case of nucleate boiling is calculated by the Jens-Lottes correlation:

$$
\Delta T_{J L}=60\left(\Phi_{c, \text { out }} / 10^{6}\right)^{0.25} \exp \left(-6.2 \times 10^{-6} p\right),
$$

with the system coolant pressure $p$ in $\mathrm{Pa}$.

The cladding temperature drop is computed by the thermal energy balance without any heat source in the annular cylinder and by Fourier law $\left(\nabla \cdot \Phi=0, \Phi=-k_{c} \nabla T\right)$; using a uniform thermal conductivity $k_{c}(\mathrm{~W} / \mathrm{m} / \mathrm{K}$, from MATPRO and valid for $T<2098 \mathrm{~K})$, it is $[8]$ :

$$
\begin{aligned}
\Delta T_{c} & =\Phi_{c, \text { out }} \frac{r_{c, \text { out }}}{k_{c}} \ln \left(\frac{r_{c, \text { out }}}{r_{c, \text { in }}}\right), \\
k_{c} & =7.51+2.09 \times 10^{-2} T-1.45 \times 10^{-5} T^{2}+7.67 \times 10^{-9} T^{3} .
\end{aligned}
$$

A likely open-gap conductance value of $h_{g}=3.40 \times 10^{3} \mathrm{~W} / \mathrm{m}^{2} / \mathrm{K}$ is assumed to estimate the temperature $T_{s}=T\left(r_{s}\right)$ at the outer surface of the pellet, with the temperature drop in the gap as $\Delta T_{g}=\Phi_{c, \text { out }} r_{c, \text { out }} /\left(r_{c, \text { in }} h_{g}\right)$. This assumption greatly eases the resolution of the thermal rod problem, thus removing the main source of non-linearity for the gap physics.

Lastly, a volume-averaged value of the fuel temperature $T_{f}$ is sought gathering further simplification for heat conduction in the pellet. Specifically, the parabolic temperature profile in the heated cylinder is used with uniform radial power distribution and constant conductivity $k_{f}(\mathrm{~W} / \mathrm{m} / \mathrm{K})$ computed at the same temperature $T_{f}$ :

$$
T(r)=T_{s}+\frac{P_{l}}{4 \pi k_{f}\left(T_{f}\right)}\left(1-\frac{r^{2}}{r_{s}^{2}}\right),
$$

whose volume average yields:

$$
T_{f}=T_{s}+\frac{P_{l}}{8 \pi k_{f}\left(T_{f}\right)} .
$$

The root of this transcendental equation provides the requested fuel temperature value. The approximation on the fuel thermal conductivity allows to solve for a linear conduction equation indeed, and serves here the simplification purpose.

These temperature values are used in the input file of the lattice transport calculations, to allow for proper interpolation of nuclear data from the evaluated data libraries. They are uniformly set in the cells of the spatial mesh.

\section{$5 \quad$ Numerical results}

The mono-dimensional coupled problem is here solved numerically with both formulations presented in section 3, based on Eq. 8 and Eq. 5. Their solutions computed with the same data of the closed channel defined in section 5.1 are then compared. 


\subsection{Preparation of nuclear data}

The diffusion coefficient and the macroscopic cross sections employed in the conservation equations are generated with several transport calculations performed by a development version of APOLLO3 ${ }^{\circledR}[9]$. They are prepared by homogenizing the calculated reaction rates on a typical UO2 pin cell of the fuel assembly $17 \times 17$ AFA 3G, used in the French 900MWe PWR unit. All design data are provided by Coppolani [10], and briefly resumed here in Tab. 1. In order to reproduce all physical conditions of the fuel cell in the channel, the calculations are performed at different moderator temperatures and linear power levels.

Table 1: Specifications of the $\mathrm{UO}_{2}$ fuel pin type used in the AFA $3 \mathrm{G}$ fuel assembly (HMM is heavy metal mass in fresh fuel).

\begin{tabular}{|r|c|l|}
\hline cell pitch & 12.588 & \multirow{2}{*}{} \\
\cline { 1 - 2 } fuel pellet diameter & 8.192 & \multirow{2}{*}{$(\mathrm{mm})$} \\
\cline { 1 - 2 } inner clad diameter & 8.528 & \\
\cline { 1 - 2 } outer clad diameter & 9.500 & \\
\hline fuel enrichment & 3.25 & $\mathrm{w} / \mathrm{o}^{235} \mathrm{U}$ \\
\hline cladding material & \multicolumn{2}{|c|}{ Zircaloy-4 } \\
\hline linear power & 178.00 & $(\mathrm{~W} / \mathrm{cm})$ \\
\hline active core height & 366.00 & $(\mathrm{~cm})$ \\
\hline channel mass flow & 334.38 & $(\mathrm{~g} / \mathrm{s})$ \\
\hline power per g of HMM & 39.70 & $(\mathrm{~W} / \mathrm{g})$ \\
\hline
\end{tabular}

The inlet and outlet temperatures of the coolant are assumed at $291^{\circ} \mathrm{C}$ and $325^{\circ} \mathrm{C}$, and using a primary pressure $p$ of 155 bar with the IAPWS-IF97 water tables [11] in the single-phase regime, this yields respectively enthalpies of 1289.42 and $1484.25(\mathrm{~kJ} / \mathrm{kg})$. Fresh fuel at beginning of cycle is modelled, with a boron dilution of 1400 PPM in the moderator.

About each transport calculation, the cross sections of ${ }^{234} \mathrm{U},{ }^{235} \mathrm{U},{ }^{238} \mathrm{U}$ and ${ }^{91} \mathrm{Zr}$ are self-shielded by the fine structure formalism (also known as the Livolant-Jeanpierre method) [12] after cylindrisation of the cell with volume conservation. After, the neutron distribution is obtained by the MOC solver* with 281 energy groups. Finally, spatial homogenization over the whole pin cell and condensation to one energy group provide the sought nuclear data for the coupled problem.

The physical data for Eqs. 8 and 11 are shown in Figure 1, confirming the trend expected from the thermal feedback. We functionalize these data by ordinary least squares regression. Tab. 2 reports the fitting coefficients of a linear fit of the kind:

$$
v=\theta_{0}+\theta_{1} \cdot x+\theta_{2} \cdot y
$$

after feature scaling and normalization, that is using the reduced variables:

$$
x=(h-\bar{h}) / \Delta_{h}, \quad y=(\phi-\bar{\phi}) / \Delta_{\phi},
$$

${ }^{*} \mathrm{MOC}$ is method of characteristics, solved by the TDT solver implemented in the APOLLO3 ${ }^{\circledR}$ code system. 
where $\Delta_{(\cdot)}$ refers to the difference between the max and the min values of the features used in the fit and (.) stands for the arithmetic mean ${ }^{\dagger}$. The fit uses 132 samples with the fuel pin calculated at BOC, and at each combination of the following linear power and moderator temperature values:

- $P_{l}=\left[i / 10 \cdot P_{l}^{N}\right.$, for $\left.i=0,1, \ldots, 11\right](\mathrm{W} / \mathrm{cm})$;

- $T_{m}=[286,291+j / \tau \cdot \Delta T, 330$, for $j=0,1, \ldots, \tau \mid \tau=8]\left({ }^{\circ} \mathrm{C}\right)$;

with $P_{l}^{N}$ as the nominal linear heat rate at $\mathrm{HFP}, P_{l}^{N}=178(\mathrm{~W} / \mathrm{cm})$, and $\Delta T=T_{o}-T_{i}$ with the inlet temperature $T_{i}=291{ }^{\circ} \mathrm{C}$ and the outlet temperature $T_{o}=325^{\circ} \mathrm{C}$. Again, primary pressure is considered as fixed to 155 bar. The total neutron flux corresponding to the simulated conditions is plotted in Fig. 2.
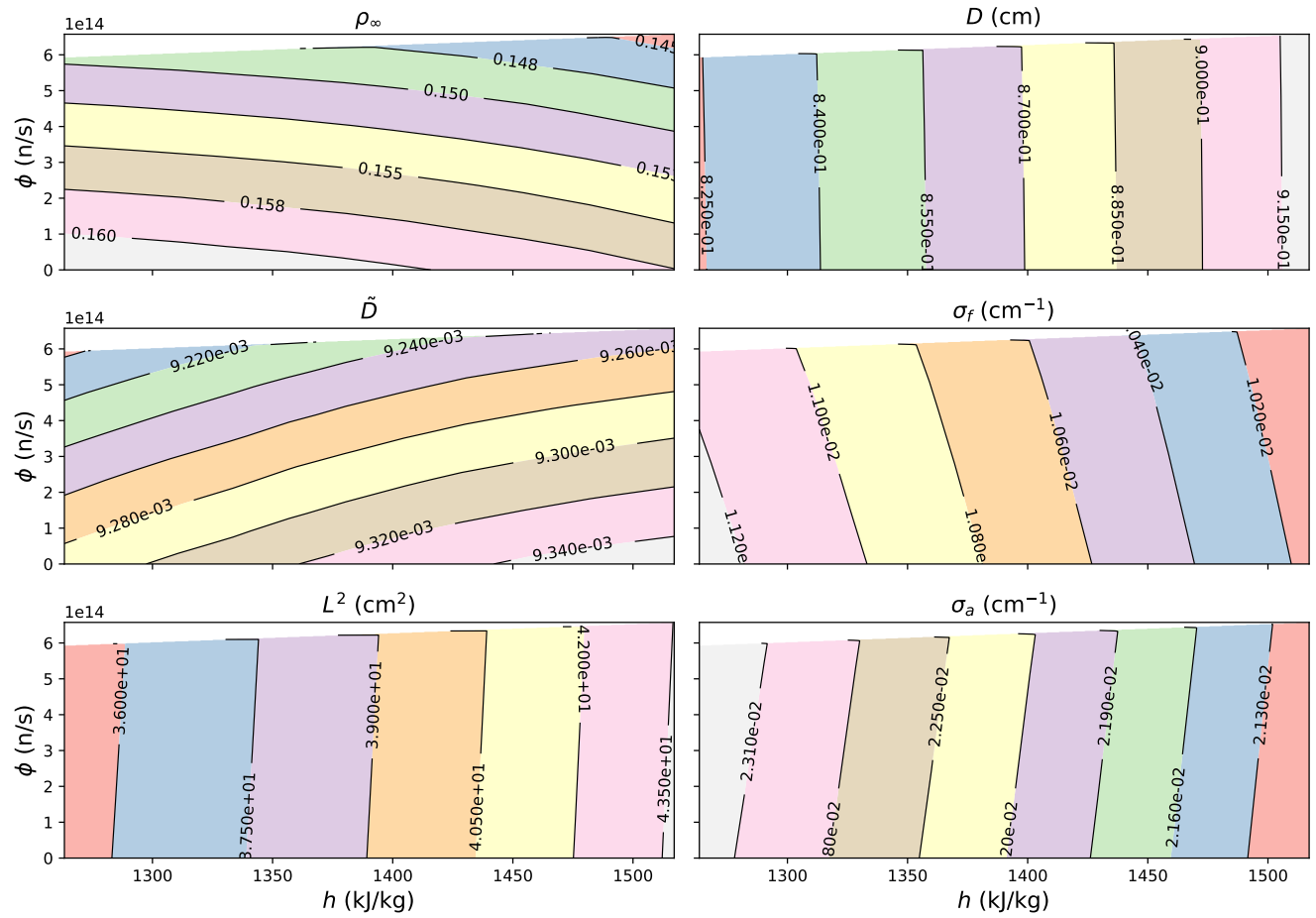

Figure 1: One group nuclear data homogenized in the fuel pin cell, as functions of the coolant enthalpy $h$ and the neutron flux $\phi$.

The additional non-linearity introduced by these functions prevents in general to find analytical solutions as $\phi(h, \rho)$ with a parametric dependence on the neutron reactivity. A simple case offering an analytical solution is presented in appendix A. Its solution can be used as initial guess for iterative techniques using some linearisation on Eq. 8. In any case, the system reactivity has to be determined as root of Eq. 10. The reason behind this approximated form of the original equation comes from the dimensionless quantity $\tilde{D}$, which changes of only a few percents over the tested range of values.

\footnotetext{
${ }^{\dagger}$ This holds for all features but the intercept, and it is required for their very different scales.
} 
Table 2: Fitting coefficients $\theta$-s determined by ordinary least square regression on one-group nuclear data; the arithmetic mean and the standard deviation of the error are available in percent as $\bar{e}$ and $\sigma_{e}$, respectively. Features are reduced with $\Delta_{h}=253.805$ and $\bar{h}=1385.197(\mathrm{~kJ} / \mathrm{kg}), \Delta_{\phi}=6.57426 e+14$ and $\bar{\phi}=3.09667 e+14(\mathrm{n} / \mathrm{s}) . \nu=2.458$ and $\kappa=198.959 \mathrm{Mev} /$ fiss.

\begin{tabular}{|c|c|c|c|c|c|c|}
\hline & $\theta_{0}$ & $\theta_{1}$ & $\theta_{2}$ & $R^{2}$ & $\bar{e}$ & $\sigma_{e}$ \\
\hline \hline$D$ & $8.67107 \mathrm{e}-01$ & $9.53613 \mathrm{e}-02$ & $5.09408 \mathrm{e}-04$ & 0.99598 & $-2.341 \mathrm{e}-04$ & 0.215 \\
$\rho_{\infty}$ & $1.54041 \mathrm{e}-01$ & $-4.15553 \mathrm{e}-03$ & $-1.35487 \mathrm{e}-02$ & 0.99641 & $-3.224 \mathrm{e}-04$ & 0.168 \\
$\sigma_{a}$ & $2.23081 \mathrm{e}-02$ & $-2.15623 \mathrm{e}-03$ & $1.06451 \mathrm{e}-04$ & 0.99832 & $-2.519 \mathrm{e}-04$ & 0.123 \\
$\sigma_{f}$ & $1.07106 \mathrm{e}-02$ & $-1.09602 \mathrm{e}-03$ & $-1.21641 \mathrm{e}-04$ & 0.99792 & $-3.502 \mathrm{e}-04$ & 0.148 \\
$\tilde{D}$ & $9.27716 \mathrm{e}-03$ & $6.45038 \mathrm{e}-05$ & $-1.00213 \mathrm{e}-04$ & 0.99281 & $-1.048 \mathrm{e}-05$ & 0.032 \\
\hline
\end{tabular}

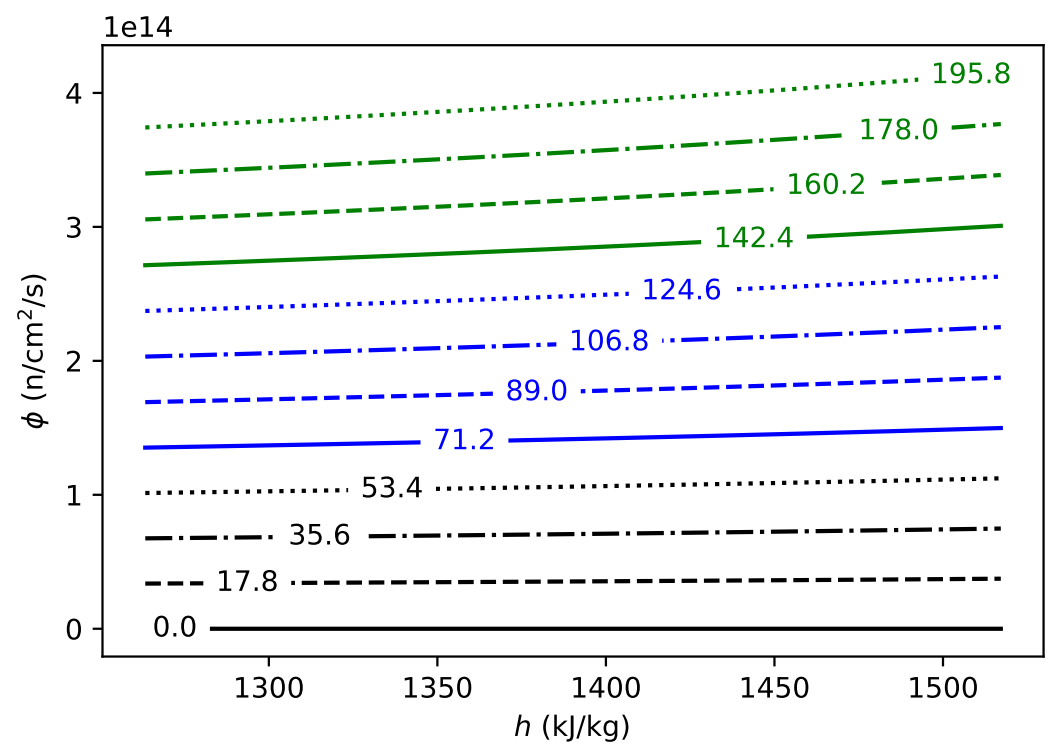

Figure 2: Total neutron flux $\phi$ computed at different combinations of the enthalpy of the moderator $h$ and of the power heat rate $P_{l}$, whose values in $\mathrm{W} / \mathrm{cm}$ are inline. 


\subsection{Numerical solution of the non-linear problem}

The numerical schemes of the two formulations solving the coupled problem are detailed in this section. Both uses the technique of successive substitutions to resolve their nonlinearities. That is the non-linear terms are linearized provided a best-guessed solution from the previous iteration and the new approximation of the solution will serve for the linearization at the next step. The new formulation resolving the flux in the enthalpy variable embeds the non-linearity in the neutron equation, taking advantage of the fact that the coefficients of the equation, i.e. the diffusion constant and the cross sections, are all defined as functions of the enthalpy and of the flux itself. Instead, the substitution of the same functional dependencies into the original diffusion equation in $z$ is not worthy since any consequent linearisation would use at least the enthalpy approximation from the previous call to the coolant equation. This demands necessarily to resolve the coupled equations separately with successive updates of the unknowns through iterations, like in the operator-splitting solving technique explained hereafter. Lately, other techniques to resolve the multi-physics coupled problems have been documented to cope with the stability and slow convergence properties of operator-splitting. However, we choose this technique in the present work to comply with the current practice in nuclear industry to simulate the reactors by computer codes.

The derivative of the diffusion Eq. 5 in the $i$-th node is discretized by central finite differences:

$$
\begin{aligned}
& -\left.d_{z}\left(D d_{z} \phi\right)\right|_{i} \approx-2 \frac{\left.\left(D d_{z} \phi\right)\right|_{i+1 / 2}-\left.\left(D d_{z} \phi\right)\right|_{i+1 / 2}}{\Delta_{i+1}+\Delta_{i}} \\
& \approx-\frac{2}{\Delta_{i+1}+\Delta_{i}}\left[\frac{D_{i+1 / 2}}{\Delta_{i+1}} \phi_{i+1}-\left(\frac{D_{i+1 / 2}}{\Delta_{i+1}}+\frac{D_{i-1 / 2}}{\Delta_{i}}\right) \phi_{i}+\frac{D_{i-1 / 2}}{\Delta_{i}} \phi_{i-1}\right] \\
& \quad \text { with } D_{i+1 / 2}=\frac{D_{i+1}+D_{i}}{2}
\end{aligned}
$$

for $i=1, \ldots, I-1$. The volume-averaged diffusion constant is set at the cell midpoints. An equidistant axial mesh of $I$ intervals is here used, therefore the central differences yields a second-order approximation. The equations for the zero incoming current boundary conditions (also known as vacuum) are derived from $\left.d_{z} \phi\right|_{i / o}= \pm \phi /\left.(2 D)\right|_{i / o}$ at inlet and outlet, respectively as:

$$
\frac{\phi_{1}-\phi_{0}}{\Delta_{1}} \approx \frac{\phi_{0}}{2 D_{0}} \text { and } \frac{\phi_{I-1}-\phi_{I}}{\Delta_{I}} \approx \frac{\phi_{I}}{2 D_{I}}
$$

The reflective boundaries are simply approximated by $\phi_{0}=\phi_{1}$ or $\phi_{I-1}=\phi_{I}$. These conditions are only first order approximations.

The coefficients from the discretization are casted in the tridiagonal matrix $\mathbf{A}$. The sum of the absorption cross section on the main diagonal makes the matrix diagonally dominant, and one can use the Thomas algorithm to solve efficiently the matrix system equations, in about $O(I)$ operations [13]. The critical problem is resolved by the power method:

$$
\mathbf{A} \vec{\phi}^{(n+1)}=\frac{1}{k^{(n)}} \nu \mathbf{F} \vec{\phi}^{(n)}, k^{(n+1)}=k^{(n)} \frac{\vec{\phi}^{(n+1)} \cdot \mathbf{F} \vec{\phi}^{(n+1)}}{\vec{\phi}^{(n+1)} \cdot \mathbf{F} \vec{\phi}^{(n)}},
$$

with the diagonal production matrix $\mathbf{F}=\operatorname{diag}\left(\overrightarrow{\sigma_{f}}\right)$ and $\overrightarrow{\sigma_{f}}=\left[\sigma_{f, i}, i=1, \ldots, I-1\right]$. The flux vector $\vec{\phi}$ always undergoes the normalization to fulfill the total power production in the channel: $\vec{\phi} \leftarrow \phi\left[\mathcal{P} /\left(\kappa \overrightarrow{\sigma_{f}} \cdot \vec{\phi}\right)\right]$. 
Integration by the trapezoidal rule on the $i$-th interval of Eq. 1 yields the enthalpy in the $(i+1)$-th node:

$$
h_{i+1}=h_{\mathrm{i}}+\frac{\kappa}{2 q}\left(\sigma_{f, i+1} \phi_{i+1}+\sigma_{f, i} \phi_{i}\right)\left(z_{i+1}-z_{i}\right),
$$

for $i=1, \ldots, I$ and $h\left(z_{i}\right)=h_{\mathrm{i}}$ as known input data. As well, the flux normalization must always verify $h\left(z_{o}\right)=h_{\mathrm{O}}$. This numerical integration is expected to underestimate the true value since the thermal power has always negative concavity upon the whole domain.

The cross sections and the diffusion constant are written as functions of the local enthalpy and flux according to Eqs. 12, see Tab. 2. Under-relaxation is mandatory to stabilize the coupling. Using the new iteration index $m$ to represent the numerical solutions obtained by Eqs. 15 and 16, this means:

$$
\sigma \leftarrow \omega \sigma\left(\phi^{(m+1)}, h^{(m+1)}\right)+(1-\omega) \sigma\left(\phi^{(m)}, h^{(m)}\right),
$$

where $\sigma$ stands generally for $D, \sigma_{a}$ and $\sigma_{f}$. The unique damping parameter of $\omega=0.495$ is used on all coefficients not to bias the neutron balance. This value results as optimal from extensive numerical tests, in absence of a stronger theoretical demonstration about the spectral radius of the global operator. Further relaxation on the flux and enthalpy is discouraged to avoid the additional burden of finding the optimal settings, possibly also ending in false convergence issues. Iterations stop when the residuals between the new and old estimates at the $m$-th and $(m-1)$-th iterations fall below the target tolerances ${ }^{\ddagger}$.

As already mentioned, the new formulation combines the coupled equations in a unique equation, Eq. 8, where the function approximations of the nuclear data is exploited in the discretized form too. Moreover, the dependence of these coefficients on the unknowns of the problem can be simplified further in order to obtain an analytical solution used as initial guess, see appendix A. This is expected to improve stability significantly, and to approach faster the fixed point solution.

A change of variable for the flux is convenient after noticing the very different scales in magnitude of the quantities in Eq. 8. So we solve for $\phi \leftarrow \phi(\kappa / q)$, since the flux is $O(q / \kappa)$. We discretize similarly the streaming term to then resolve the following equation:

$$
\begin{aligned}
&-\left.d_{h}\left(\tilde{D} d_{h} \phi^{2}\right)\right|_{i} \approx-2 \frac{\left.\left(\tilde{D} d_{h} \phi^{2}\right)\right|_{i+1 / 2}-\left.\left(\tilde{D} d_{h} \phi^{2}\right)\right|_{i+1 / 2}}{\Delta_{i+1}+\Delta_{i}} \\
& \approx-\frac{2}{\Delta_{i+1}+\Delta_{i}}\left[\frac{\tilde{D}_{i+1 / 2}}{\Delta_{i+1}} \phi_{i+1}^{2}-\left(\frac{\tilde{D}_{i+1 / 2}}{\Delta_{i+1}}+\frac{\tilde{D}_{i-1 / 2}}{\Delta_{i}}\right) \phi_{i}+\frac{\tilde{D}_{i-1 / 2}}{\Delta_{i}} \phi_{i-1}^{2}\right] \\
& \quad=\zeta\left(\rho_{\infty, i}-\rho\right) \text { with } \tilde{D}_{i+1 / 2}=\frac{\tilde{D}_{i+1}+\tilde{D}_{i}}{2}
\end{aligned}
$$

for $i=1, \ldots, I-1$, and again with the volume-averaged pseudo-diffusion constants at the midpoint of the intervals. The coefficients in Eq. 17 are now replaced by the linear expressions of the kind $\sigma_{i}=\theta_{0}+\theta_{1} h_{i}+\theta_{2} \phi_{i}, i=0, \ldots, I$, with the constants $\theta$ 's from Tab. 2. For the numerical solution, the flux dependence in each $\tilde{D}_{i}$ is given by the last estimates from the previous iteration, say $(m-1)$, whereas the corresponding term of $\rho_{\infty}$ is moved to the LHS and modified as $\left(\zeta \theta_{\rho_{\infty}, 2} / \phi_{i}^{(m-1)}\right)$ along the main diagonal of the

$\ddagger$ Tolerances of 1.E-7 are used in our numerical experiments for all relative residuals, including flux, enthalpy and cross section data. 
new tridiagonal matrix. This ensures diagonal dominance, allowing for new calls to the Thomas algorithm. Also, the source terms of the system equations at RHS do not need any update while iterating. The vacuum boundary conditions become:

$$
\frac{\phi_{1}^{2,(m)}-\phi_{0}^{2,(m)}}{\Delta_{1}} \approx \frac{\phi_{0}^{2,(m)}}{\tilde{D}_{0} \phi_{0}^{(m-1)}} \text { and } \frac{\phi_{I-1}^{2,(m)}-\phi_{I}^{2,(m)}}{\Delta_{I}} \approx \frac{\phi_{I}^{2,(m)}}{\tilde{D}_{I} \phi_{I}^{(m-1)}},
$$

requesting the same kind of update. The squared flux is readily forced to be equal at the two neighboring points close to the boundary in case of reflection, as above. These modifications are justified by recognizing the squared flux as the new unknown. For sake of completeness, we remind that zero flux at the boundary does not need any additional closure relation. The rank of the matrix $\mathbf{A}$ is always $(I-1)$.

In substance, the new formulation obtains first the solution on the enthalpy mesh, which is reverted after onto the $z$ axial mesh, still by means of Eq. 16. Instead, the classical diffusion equation provides already the solution on the axial coordinate, but requires to solve Eq. 1 many times in order to get new enthalpy distributions and cross sections update. Notably, Eq. 5 implies the resolution of an eigenvalue problem which is computationally more expensive than a fixed source problem.

Integration of the inverse power in Eq. 11 requires to handle carefully the boundary with zero flux. If a primitive function was possible, the integration would be resolved by means of an improper integral, since we known that the power is produced within a finite active length. Numerical shrewdness is compelling to treat this integration on the boundary cells also in case of vacuum, that shows steep flux gradients too. According to appendix A, the flux behaves for instance like $h \sqrt{h}$ at inlet. Therefore, the simple trapezoidal rule is not recommended for the integration of the inverse power. The quadratic Simpson's rule could be an alternative, but arrangements would still be needed at these boundaries for the manifest loss of smoothness. Eventually, rational splines could be a valid option. Here, we use Eq. 16 with both formulations, accepting to deal with finer meshes to increase the accuracy. Consistently, when the difference $\left(z_{i+1}-z_{i}\right)$ is to be determined, Eq. 16 is divided by the average power in the $(i+1)$-th cell.

About Eq. 16, equidistant axial meshes in enthalpy may generate high error where the power is lower, that is near boundaries in our problem. Hence, we refine the enthalpy mesh at the boundaries according to the formula:

$$
h_{i}=\frac{1}{2}\left[\left(h_{\mathrm{O}}+h_{\mathrm{i}}\right)-\left(h_{\mathrm{O}}-h_{\mathrm{i}}\right) \cos \left(\frac{h_{i}-h_{\mathrm{i}}}{h_{\mathrm{O}}-h_{\mathrm{i}}} \pi\right)\right] .
$$

Apart from the numerical errors, the solutions of the two formulations can differ because they employ different coefficients, with their own given functions indeed. For instance differences arise yet when recalculating $\rho_{\infty}$ by the standard one-group definition, see Fig. 3. A smart redefinition of these coefficients is suggested to verify and to compare their results. In fact, all data can be determined by $\sigma_{f}, \tilde{D}$ and $\rho_{\infty}$ :

$$
D=\tilde{D} / \sigma_{f}, \sigma_{a}=\left(1-\rho_{\infty}\right) \nu \sigma_{f}
$$

still provided the constant neutron multiplicity $\nu$. A verification against an analytical solution is then possible using the problem in appendix A.

Both solving techniques need iterations to update sequentially the coefficients of their neutron balance equation. Because of criticality, there are at least two possible levels of iterations. The former level looks for the couple of critical parameter (the reactivity) and 


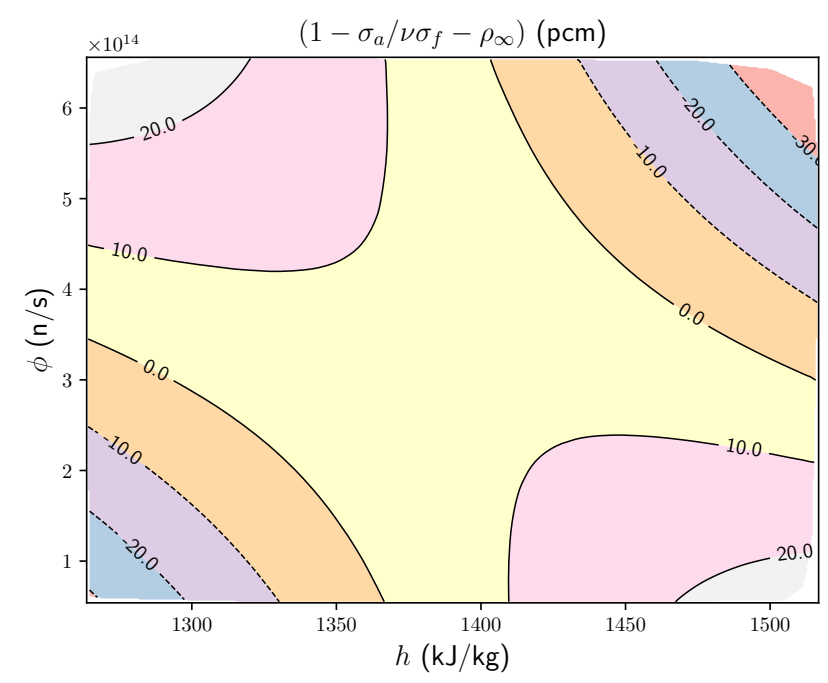

Figure 3: Differences between the fitted $\rho_{\infty}$ and the values computed by using $\rho_{\infty}=1-1 / k_{\infty}$, with $k_{\infty}=\nu \sigma_{f} / \sigma_{a}$.

fundamental flux ${ }^{\S}$. The latter level finds the right match between the neutron flux and the coolant enthalpy all along the active height of the channel, resulting in the final cross section data satisfying the neutron equation. The order of the iteration levels is reversed with the new formulation, retrieving first the flux as a function of enthalpy and then finding the reactivity as a root of Eq. 11. In any case, iterations for criticality always require to solve the neutron equation at each step, but the new formulation avoids to recur to eigenvalue problems.

The two methods introduced so far were implemented in a Python2.7 module delegating all numerical calculations to the Numpy v1.14.0 package, offering high-performance array manipulation. The use of Python is also motivated by the educational purpose of this work, aiming to a quick comprehension of the code. The results in the channel with zero incoming current boundary conditions at both inlet and outlet are presented in the following.

The convergence rates of the main unknowns are plotted in Fig. 5 for increasing numbers of spatial elements up to $I=5000$ (bottom plot). These rates are determined with the results computed by the new formulation based on the non-linear diffusive solver, confirming the expected (almost) second-order trend. Maximum relative differences between the solutions obtained by the two formulations are shown in the figure on the top. They are due to the different spatial meshes used for the calculations, always being equi-spaced when solving in $z$ and cosine-distributed for the non-linear solver in $h$. These differences fall very fast, suggesting meshes of the order of a few centimeters when comparing the methods. A number of cells $I=100$ is already enough to get reactivity differences below the $\mathrm{pcm}$ and power differences lower than $0.1 \%$. The corresponding differences between the computed cross section data are reported in Fig. 6 . The integration by the trapezoidal rule should be changed with a higher order method if more accurate approximations of the

\footnotetext{
$\S_{\text {This }}$ couple becomes an eigenpair of a generalized eigenvalue problem with Eq. 5.
} 
steeper power gradients towards the boundaries are requested. This is expected to reduce the observed fluctuations in the same figure. Finally, the axial profiles of the computed data follow in Fig. 7. As anticipated by the thermal feedback on the cross sections, the power is peaked in the lower part of the channel, where the coolant is colder and denser, favoring neutron thermalization. The search of the critical reactivity $\rho_{c}$ corresponding to the target active core height $H_{T}$ is plotted in Fig. 4. The neutron reactivity increases with $H$ since $\Delta H=H_{T}-H(\rho)$, approaching asymptotically the limit of the infinite medium (see $\theta_{0}$ of $\rho_{\infty}$ ). Monotone trends are so observed for both the reactivity and the flux distribution, tending to a vanishing flux in longer channels to fulfill the given power constraint. Conversely, more neutrons are needed in shorter channels.
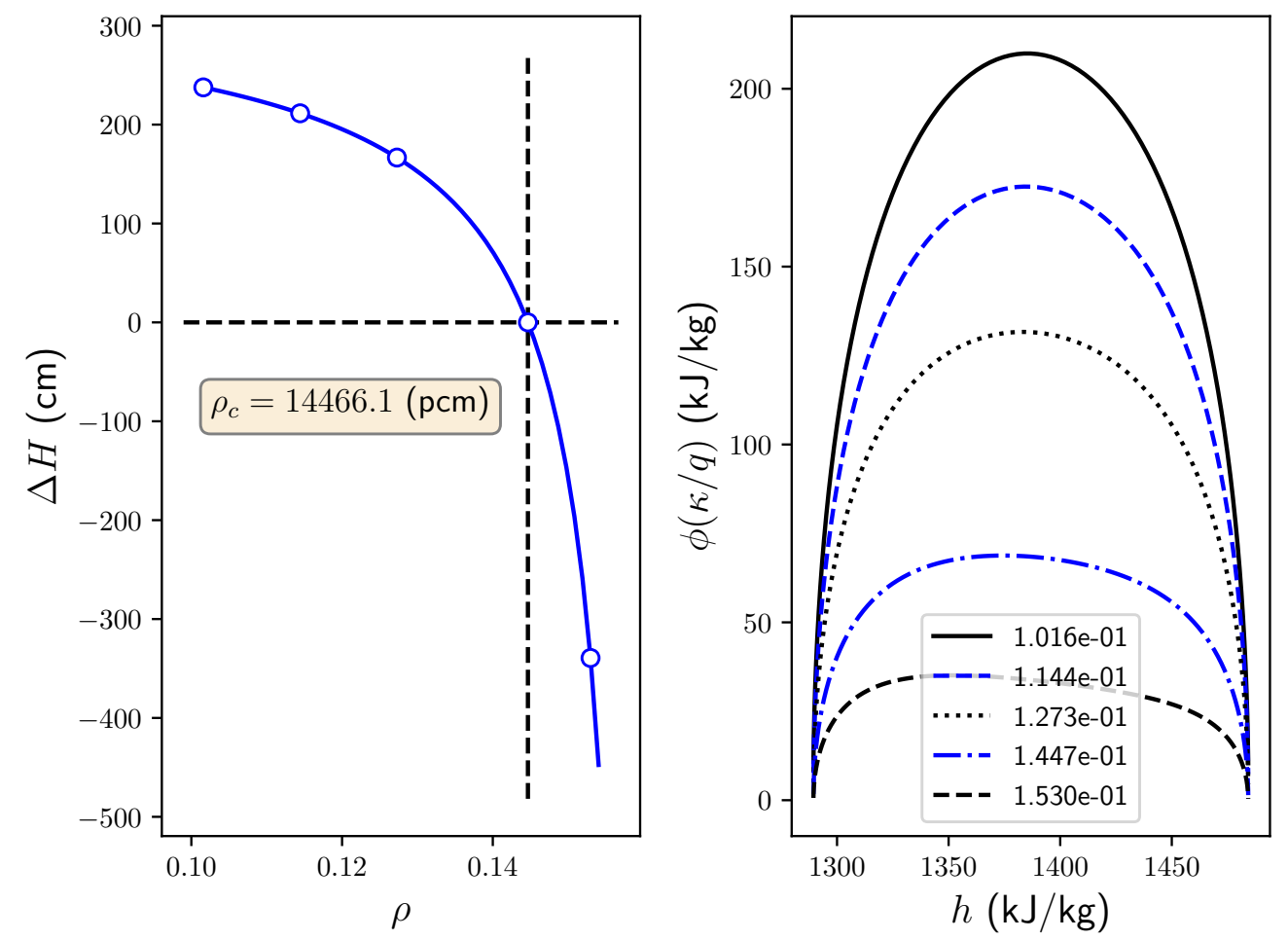

Figure 4: Search of the critical reactivity (left) with the flux distributions (right) corresponding to the states marked by the empty dots.

The supercriticality of the channel is due to a low boron content set in water when preparing the cross sections by the lattice calculations. The use of fresh fuel usually requires strong concentrations of neutron absorbers, either diluted in the coolant or mixed in the fuel, to control and operate the reactor.

Although the problems were solved through an interpreted language which is formally preventing detailed estimates of runtime performances, much shorter calculations were noticed when choosing the non-linear solver. The search of the root reactivity can be included in the iterative solver itself, without any extra cost for several eigenvalue problems to resolve. 

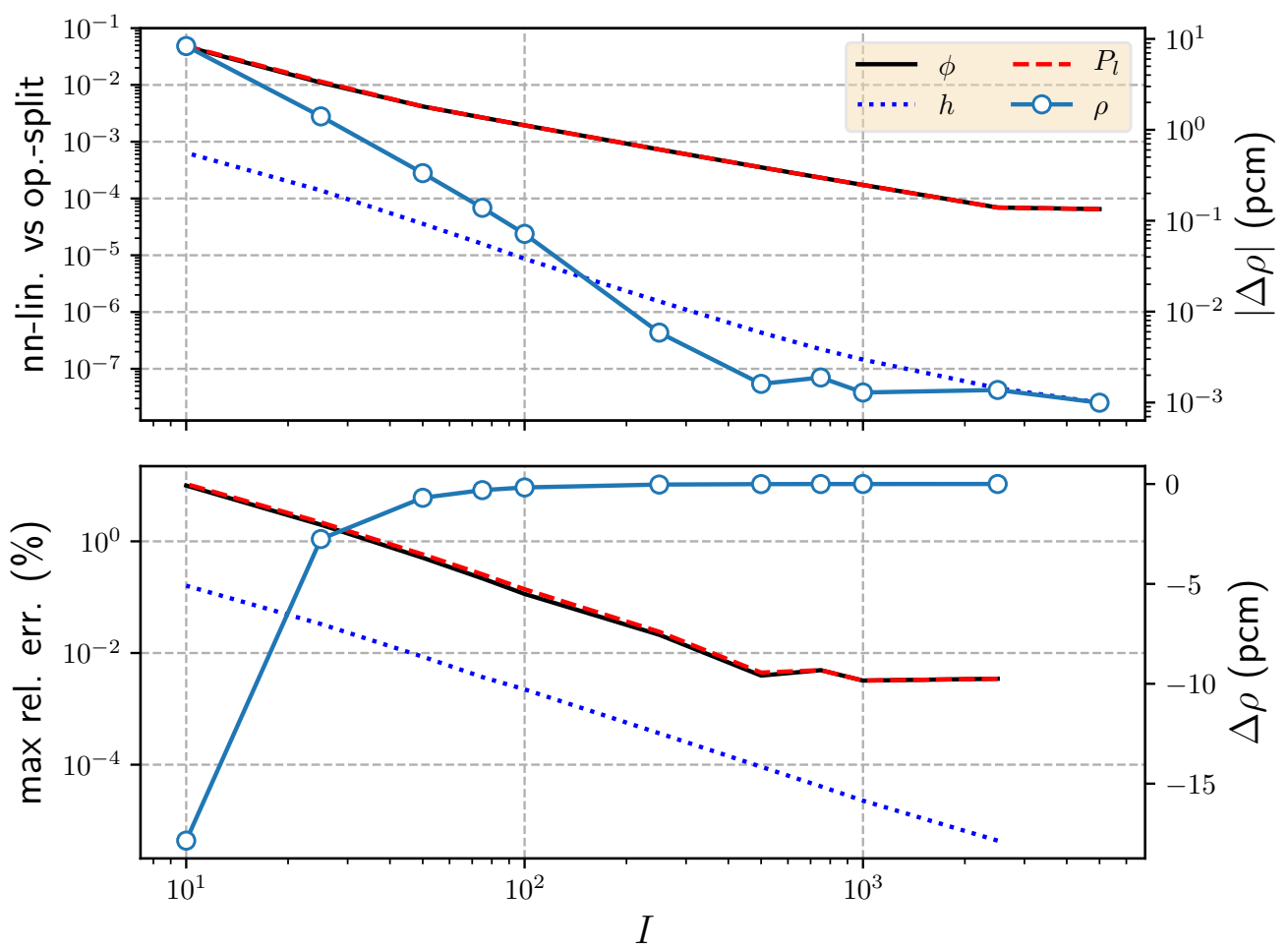

Figure 5: Maximum relative differences between the solutions computed by the two formulations (top) and convergence rates of the quantities calculated by the new formulation based on the non-linear diffusion equation in $h$. 

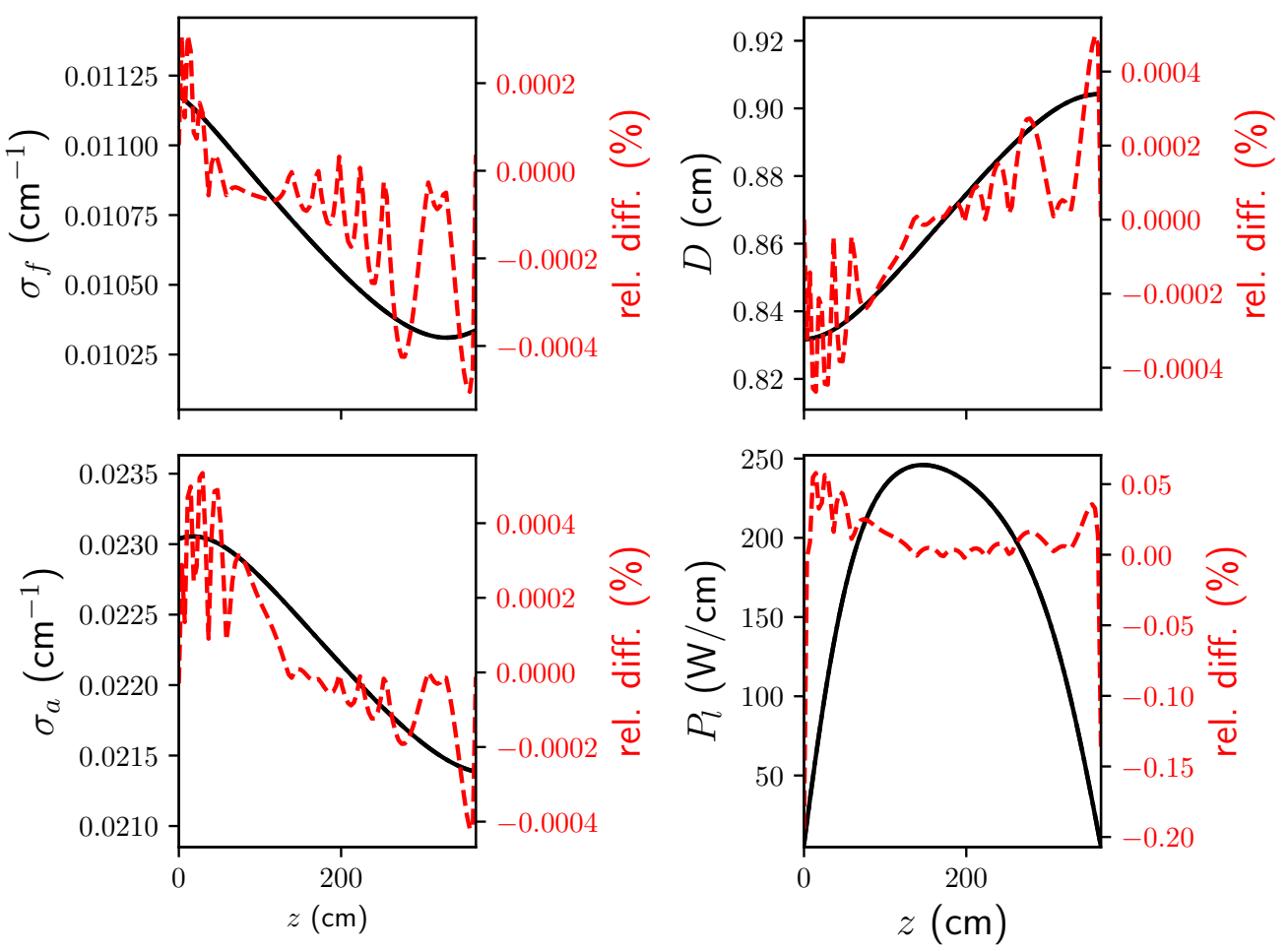

Figure 6: Differences between the cross sections calculated by the two formulations with $I=100$. 


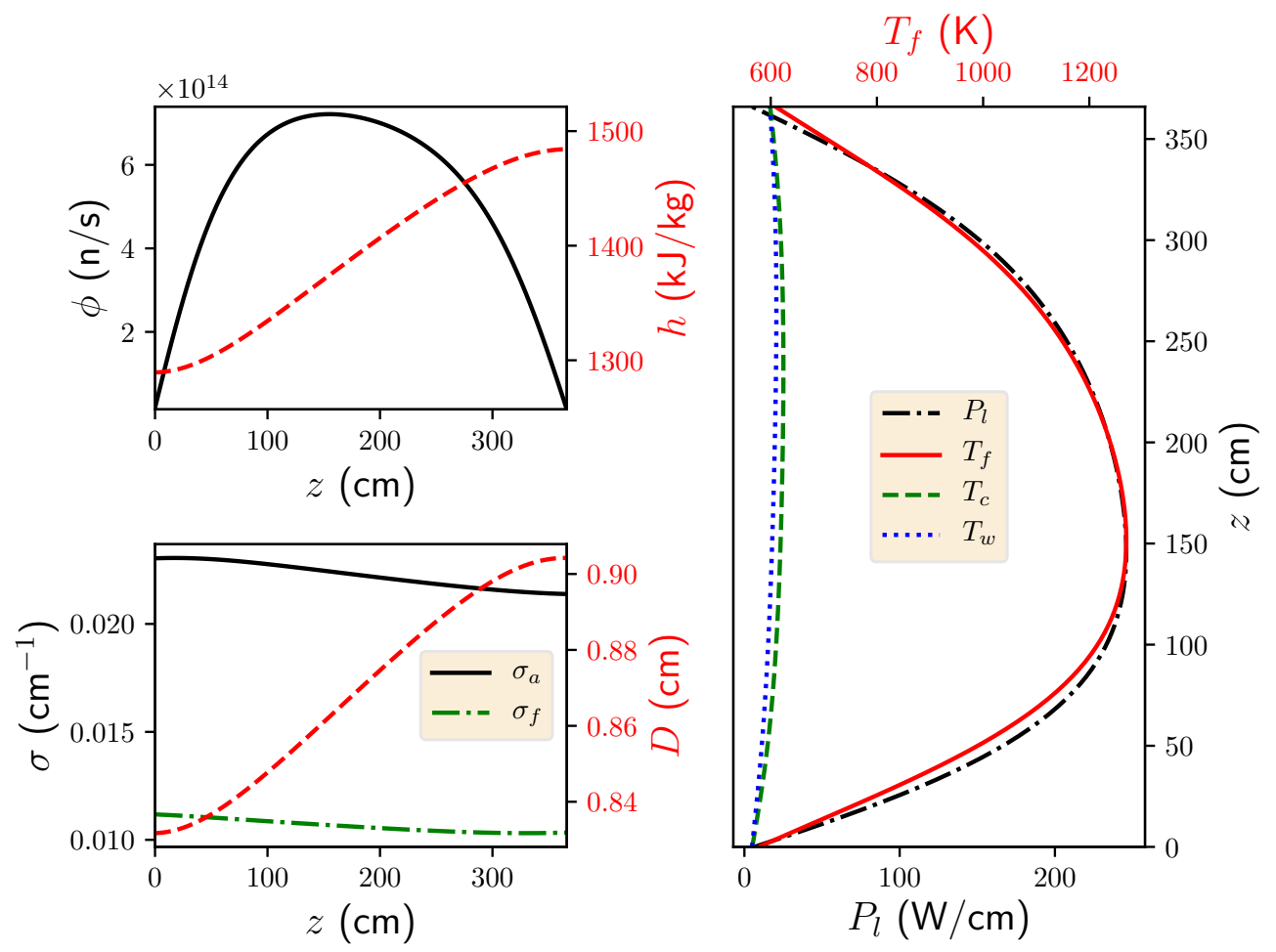

Figure 7: Axial distributions of the main quantities of interest, computed by the new formulation with $I=100$. 


\section{Conclusion}

The closed channel is a fundamental model to study the multiphysics coupling between neutronics and thermo-hydraulics. It offers a solid frame for both research and educational programs. In this preliminary work, a new formulation to resolve this coupled problem is explored with its simplest version, that is using incompressible flow without any dissipative friction, and one-group neutron diffusion theory.

This technique is actually suggested by the fact that the coefficients of the coupled equations are generally pure functions of the main unknowns. Hence, the functional dependencies are recovered in a new equation with the goal to determine the neutron flux as a pure function of the coolant enthalpy. A change of variable is at the base of this new derivation, and it holds as far as there is thermal power generated by fission in the channel. Non-fissile regions, like reflectors, require instead additional dedicated models to reproduce their influence on the active part of the channel. This means de facto a different problem definition, to be addressed in future works. The intended solutions along the axis of the channel is obtained by searching for the critical reactivity, which allows to match the target power constrained to the given active height of the core.

This new formulation is discussed and demonstrated on a subchannel problem, derived from a regular UO2 fuel pin cell typical of PWR. Its performance and results are compared to the traditional solving technique based on operator-splitting and successive substitutions, which is the most used in the nuclear industry. This last does not change the original equations to find the fixed point solution, with the advantage of using general computer codes specifically developed to resolve a single physics. However, its resolution of the coupled problem can not avoid several calls to expensive eigenvalue solvers used in reactor physics for the neutron balance. Noticeably, the new technique does not share this drawback, also embedding the possible non-linearities of the cross sections functionalization in a unique equation.

The preparation of the cross sections data is illustrated in order to support the numerical test. A new parameterization is advanced in opposition to the common use of the temperature to characterize the physical states of the fuel and of the moderator. A simple thermal model is then described to explain the relation between the radial temperature profile in the fuel rod, the coolant enthalpy and the generated power.

Despite the simplicity of the coupled problem, the current technique can be proposed as a preconditioner for the more complex tri-dimensional problems of core design. Extension to the multi-group neutron formalism and the integration of more realistic hydraulic models are advocated for future development.

\section{Acknowledgement}

The author would like to thank Dr. Emiliano Masiello for his constructive criticism of this work.

\section{A Simplified model without Doppler feedback}

A simplified model derived from Eq. 8 is resolved analytically in this section. The solution is used in this work as initial guess of the iterative technique solving the original non-linear coupled problem. 
The diffusion constant and the fission cross section are assumed constant over the all active height, whereas the dependence of the infinite reactivity on the neutron flux is dropped. These simplifications disregard the Doppler feedback of the fuel temperature on the neutron flux (and generated thermal power). But they are also aimed to obtain an analytical solution after recovering a linear equation by the substitution $u=\phi^{2}$ :

$$
\begin{array}{r}
d_{h}^{2} u=a_{0}+a_{1} h, \text { with } \\
a_{0}=\frac{\zeta}{\tilde{D}}\left(\rho-c_{0}+c_{1} \frac{\bar{h}}{\Delta_{h}}\right), a_{1}=-\frac{\zeta}{\Delta_{h}} c_{1},
\end{array}
$$

for $\rho_{\infty}=c_{0}+c_{1}(h-\bar{h}) / \Delta_{h}$. This assumption is motivated by the dimensionless quantity $\tilde{D}$ that is almost constant over the tested range of values.

The solution of the linear ODE is in the form:

$$
u=C_{1}+C_{2} h+\frac{1}{2} a_{0} h^{2}+\frac{1}{6} a_{1} h^{3},
$$

with the two real constants $C_{1}$ and $C_{2}$ by fulfilling the boundary conditions. It must be noted that the same modulus of the flux derivative in enthalpy at both inlet and outlet in case of zero incoming current eases the calculation of the constants, see Eq. 9. It must be noted that the solution does not exist if reflection is applied at both boundaries, unless $c_{1}=0$. As an example, the flux distribution corresponding to the channel problem outlined in section 5.1 and computed with the data from Tab. 2 is plotted in Fig. 8. Zero flux boundary conditions are intentionally set to show the cusp singularities, that do not prevent however to retrieve the solution in $z$.

The need of a fully positive solution for $u$ in a purely multiplying medium may demand formally for $a_{0}+a_{1} h \leq 0$ at any position hosting a non-vanishing neutron flux. At the same time, the sign of the derivative at the bottom and at the top of the channel is also specified by the physics of the problem, thus requiring that: $\left(a_{0} h_{\mathrm{i}}+1 / 2 a_{1} h_{\mathrm{i}}^{2}\right)>-C_{2}>$ $\left(a_{0} h_{\mathrm{O}}+1 / 2 a_{1} h_{\mathrm{O}}^{2}\right)$.

Finally, we only keep the positive root $\phi=\sqrt{u}$ as physical solution. The system reactivity is obtained numerically as root of Eq. 10. The square root introduced when retrieving the solution of the neutron flux may not allow in general an analytical solution for the primitive of the integral.

Eq. 20 becomes a parametric Poisson equation if $c_{1}=0$. As well, the infinite medium is reproduced by the vanishing curvature of the flux in all points of the domain, implying necessarily $\rho=c_{0}=\rho_{\infty}$. Still with $c_{1}=0$ and when both boundary conditions are not of the reflection type, the reactivity must be sought in the semi-plane $\rho<c_{0}$.

\section{References}

[1] Kostadin Ivanov and Maria Avramova. Challenges in coupled thermal-hydraulics and neutronics simulations for LWR safety analysis. Annals of Nuclear Energy, 34(6):501$513,2007$.

[2] Stéphane Dellacherie, Erell Jamelot, and Olivier Lafitte. A simple monodimensional model coupling an enthalpy transport equation and a neutron diffusion equation. Applied Mathematics Letters, 62:35-41, 2016.

[3] Stéphane Dellacherie and Olivier Lafitte. Une solution explicite monodimensionnelle d'un modèle simplifié de couplage stationnaire thermohydraulique-neutronique. $A n$ nales mathématiques du Québec, 41(2):221-264, 2017. in French. 


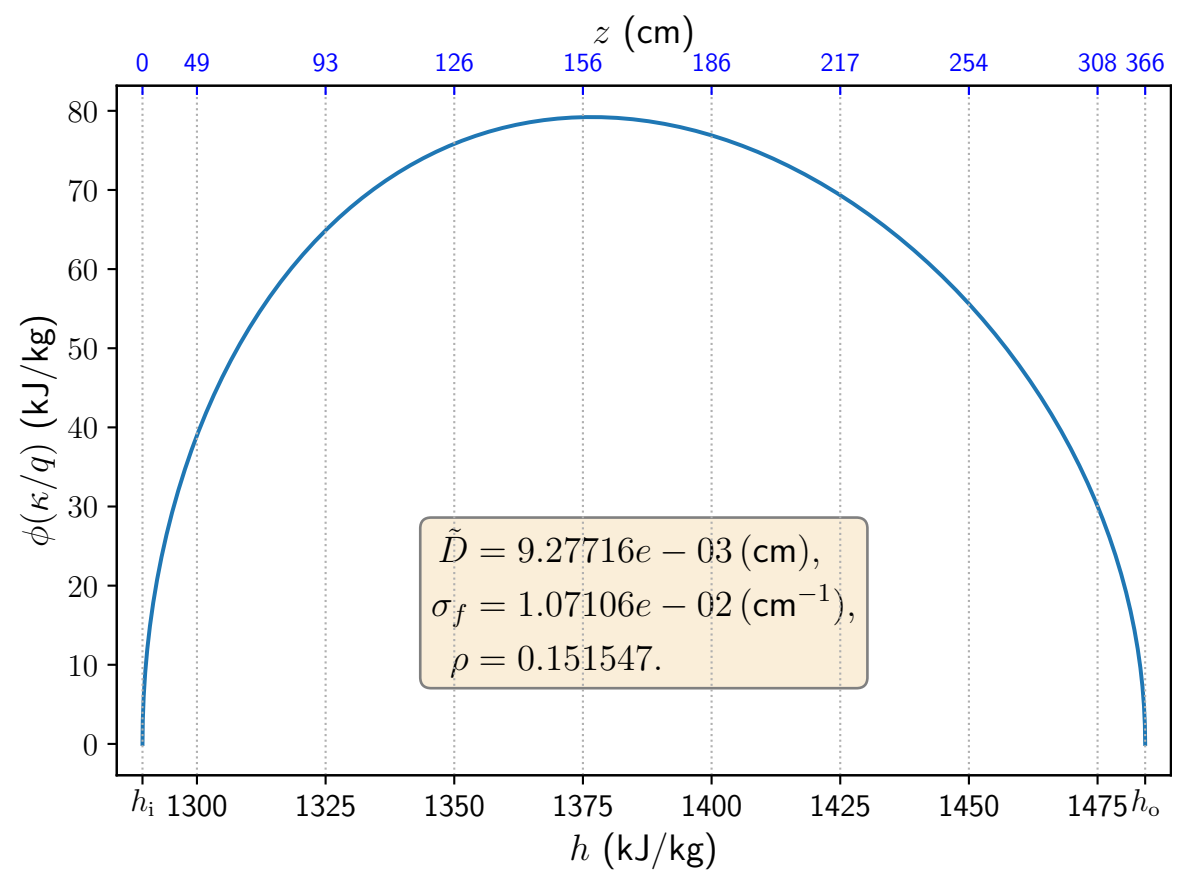

Figure 8: Analytical solution with zero flux boundary conditions at both inlet and outlet. 
[4] Stéphane Dellacherie, Erell Jamelot, Olivier Lafitte, and Riyaz Mouhamad. Numerical Results for the Coupling of a Simple Neutronics Diffusion Model and a Simple Hydrodynamics Low Mach Number Model without Coupling Codes. In Symbolic and Numeric Algorithms for Scientific Computing (SYNASC), 2016 18th International Symposium on, pages 119-124. IEEE, 2016.

[5] Neil E Todreas and Mujid S Kazimi. Nuclear systems: thermal hydraulic fundamentals, volume 1. CRC press, 2012.

[6] Robert Vartan Meghreblian and David K Holmes. Reactor Analysis. McGraw-Hill, 1960.

[7] KJ Geelhood, WG Luscher, P Raynaud, and I Porter. FRAPCON-4.0: A Computer Code for the Calculation of Steady-State. Technical Report PNNL-19418, Vol. 1 Rev. 2, Pacific Northwest National Laboratory, USA, September 2015.

[8] WG Luscher, KJ Geelhood, and I Porter. Material Property Correlations. Technical Report PNNL-19417, Rev. 2, Pacific Northwest National Laboratory, USA, September 2015 .

[9] D Schneider, F Dolci, F Gabriel, JM Palau, M Guillo, B Pothet, P Archier, K Ammar, F Auffret, R Baron, et al. APOLLO3: CEA/DEN deterministic multi-purpose code for reactor physics analysis. In Proc. Int. Conf. Physics of Reactors (PHYSOR2016), 2016 .

[10] Pierre Coppolani. La chaudière des réacteurs à eau sous pression. EDP Sciences, 2012. in French.

[11] Wolfgang Wagner and Hans-Joachim Kretzschmar. International Steam TablesProperties of Water and Steam based on the Industrial Formulation IAPWS-IF9\%. Springer Science \& Business Media, 2007.

[12] Alain Hébert. Applied Reactor Physics. Presses inter Polytechnique, 2009.

[13] Alfio Quarteroni, Riccardo Sacco, and Fausto Saleri. Numerical mathematics, volume 37. Springer Science \& Business Media, 2010.

[14] Aldo Dall'Osso and Léandre Brault. On the neutron spectrum and multiplication factor in the infinite homogeneous reactor. Annals of Nuclear Energy, 36(8):1287$1293,2009$. 